for leukemia, 93 children, aged 4-15 years, were allocated a random sequence of 4 LPs, two with a 22-guage needle and two with a 25 -gauge needle, all performed under general anesthesia (sevoflurane). A single needle insertion was used in 320 (94\%) procedures; multiple attempts were required in 21 (9 with 22-gauge needle and 12 with 25-gauge $(\mathrm{p}=0.5)$.

Analysis of 341 LPs showed an incidence of $7.2 \%$ post-LP headache that followed the use of a 22-gauge needle was not significantly different from an incidence of $4.6 \%$ when using a 25 -gauge needle $(\mathrm{p}=0.3)$. Also, the incidence of any headache following LP was not related to needle size (18\% with 22 -gauge needle and $15 \%$ with 25 gauge needle; $\mathrm{p}=0.4$ ). Having one LP headache did not predispose to recurrence; only one child had two LP headaches. LP procedure time (time for collection of 22 drops [1 $\mathrm{ml}$ CSF) was doubled when using the 25 -gauge needle. Incidence of post-LP headache was not lower in younger children and was unrelated to age; it was higher in girls than in boys $(11 \%$ vs $3 \%$, respectively, $\mathrm{p}=0.014)$. The overall functional impact of post-LP headache in a child with leukemia was assessed as moderate or severe in $55 \%$ of families. (Crock C, Orsini F, Lee KJ, Phillips RJ. Headache after lumbar puncture: randomized crossover trial of 22-gauge versus 25-gauge needles. Arch Dis Child 2014 Mar;99(3):203-7).

COMMENTARY. Incidence of post-LP headache in children treated for leukemia is higher following use of a 22-gauge compared to a 25 -gauge needle, but the difference is not significant. The authors conclude that either gauge may be appropriate for LP in a child. In contrast, adults have a significantly lower incidence of LP headache when using smaller diameter needles and needles with a blunt, pencil-type point rather than the traditional cutting point [1][2].

\title{
References.
}

1. Carson D, Serpell M. Neurology. 1996 Jul;47(1):33-7.

2. Janssens E, et al. Eur J Pediatr. 2003 Mar; 162(3):117-21.

\section{ROLE OF PACAP IN MIGRAINE HEADACHES}

Investigators at Danish Headache Centre, University of Copenhagen, Glostrup, Denmark, studied the incidence of migraine during and after intravenous infusion of pituitary adenylate cyclase-activating polypeptide-38 (PACAP38) and vasoactive intestinal polypeptide (VIP) in 22 female migraine patients without aura (mean age 24 years [range 19-36]). Sixteen patients (73\%) reported migraine-like attacks after PACAP38 and 4 after VIP (18\%) infusion ( $\mathrm{P}=0.002)$. Three of 4 patients who reported migraine-like attacks after VIP also reported attacks after PACAP38. Both peptides induced dilatation of extracranial $(\mathrm{P}<0.05)$ but not intracranial arteries $(\mathrm{P}>0.05)$. PACAP38-induced vasodilatation lasted $>2 \mathrm{~h}$ whereas VIP-induced dilatation was normalized after $2 \mathrm{~h}$. Plasma PACAP38 levels were elevated at $1 \mathrm{~h}$ after starting infusion only in patients who reported migraine attacks. PACAP38 has a high affinity for the $\mathrm{PAC} 1$ receptor. Activation of the $\mathrm{PAC1}$ receptor may explain the mechanism of migraine and offer a target for development of anti-migraine drugs. (Amin FM, Hougard A, et al. Investigation of the pathophysiological mechanisms of migraine attacks induced by pituitary adenylate cyclase-activating polypeptide-38. Brain 2014 Mar;137:779-94). 
COMMENTARY. A commentary from the Department of Neurology, University of Szeged, Hungary, discusses the trigemino-vascular theory of migraine and the paintransmission link between the vascular and neuronal regions [1]. PACAP and other neuropeptides have essential roles in activation of the trigemino-vascular system. PACAP38 is present in the trigeminal ganglion and caudal trigeminal nucleus. The effects of PACAP38 are mediated through G-protein-linked receptors, including PAC1. The clinical study by Amin et al [2] is consistent with previous laboratory animal studies comparing the effects of nitroglycerol and PACAP on the trigemino-vascular system [3].

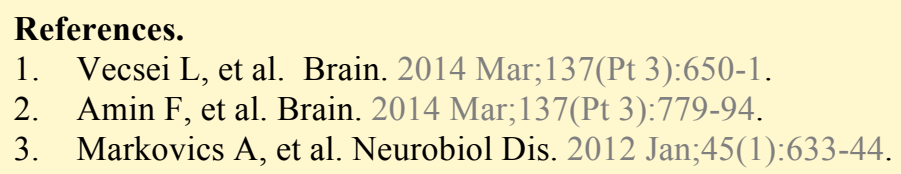

\section{NEUROMUSCULAR DISORDERS}

\section{PHARYNGEAL-CERVICAL-BRACHIAL VARIANT OF GUILLAIN- BARRE SYNDROME}

Investigators from National University Hospital, Singapore, review the clinical features of 13 cases of pharyngeal-cervical-brachial (PCB) variant of Guillain-Barre syndrome (GBS) and outline new diagnostic criteria. In a series of 100 patients with PCB reported previously from Japan [1] the age of onset ranged from 5-83 years (median age 43), antecedent upper respiratory tract infections and diarrhea occurred in $71 \%$ and $30 \%$, respectively (similar to GBS), and 31\% had serological evidence of Campylobacter jejuni infection. Cytomegalovirus, Epstein-Barr virus, Mycoplasma pneumoniae and Hemophilus influenzae were associated infrequently (6-1\%). PCB is defined as 'pure' in patients presenting with rapidly progressive oropharyngeal and cervicobrachial weakness associated with areflexia/hyporeflexia but without ophthalmoplegia or leg weakness [2]. In contrast, some recent series describe sensory disturbance in the upper limbs, and normal or exaggerated reflexes. GBS forms a continuum of overlapping syndromes, those cases with ophthalmoplegia and ataxia overlapping with Fisher syndrome. One half of patients with PCB carry IgG anti-GT1a antibodies that cross-react with GQ1b, whereas most patients with Miller Fisher syndrome carry IgG and GQ1b antibodies that always cross-react with GT1a. Significant overlap between the clinical and serological profiles of these syndromes suggests a PCB/Fisher syndrome continuous spectrum. The neurophysiological findings in PCB are axonal rather than demyelinating. Myasthenia gravis, botulism and other myopathic disorders are differentiated from PCB by the absence of sensory deficits or areflexia. (Wakerly BR, Yuki N. Pharyngeal-cervicalbrachial variant of Guillain-Barre syndrome. J Neurol Neurosurg Psychiatry 2014 Mar;85(3):339-44).

COMMENTARY. The diagnostic criteria for PCB variant of GBS include: 1) symmetric oropharyngeal weakness, arm weakness and areflexia/hyporeflexia; 2) absence of leg weakness, ataxia and disturbed consciousness; and 3) 12hr-28day interval 